\title{
Study of Motion Blurred Image Restoration Method
}

\author{
Yubing Dong, Huaxun Zhang, and Ying Sun \\ College of Electronics and Information Engineering, Changchun \\ University, Changchun, 130022, China
}

\begin{abstract}
A mathematical model of motion blur is introduced. Based on block registration, combination Wiener filter recovery method of motion blurred images is proposed. And in the MATLAB platform, the method is simulated, which gives experimental results and result analysis. The experiment shows that the method can effectively restore intelligent traffic domain with comparatively high restoration efficiency. It also has better robustness and accurate.

Keywords:image restoration, motion blur, quality evaluation, block registration.
\end{abstract}

\section{Introduction}

It is well known that during formation, transmission or recording processes, such as atmospheric turbulence, sampling, quantization, motion blur, camera misfocus, noise, etc, images are deteriorated. Degraded images often cause the loss of image quality, specific performance for image blur and deformation distortion. So the image quality seriously restrict and affect the subsequent image understanding, as well as more advanced research of target identification and target tracking. Image restoration technology is presented at the historic moment. Image restoration is the operation of taking a corrupted or noisy image and estimating the clean original image. The purpose of image restoration is to compensate for or undo defects which degrade an image.

According to the understanding of the a priori knowledge of image degradation, image restoration method can be divided into two categories. One kind is that we know types of image degradation and degradation mechanism. The degradation model can be established according to the prior knowledge. A variety of the degradation processing methods for image restoration processing are used, such as inverse filtering, wiener filtering and other classical filtering methods. Another kind is that we do not know a priori knowledge of recovery. We do not have any information about the blurring function or on the additive noise. So many estimates and iteration methods are used to recover, such as classical iterative blind de-convolution method. Because of the complexity of the image restoration research, there is still no a general image restoration method. It is very necessary to continue to carry out in-depth research of image restoration. 
The paper is organized as follows. In the next section, we propose the restoration algorithm that we research in this paper, and degradation model and estimating the degradation function are given. An image restoration method based on block registration is presented. In Section 3, evaluation criterion of recovery quality is introduced. Section 4 presents experimental results using kinds of restoration methods. And the computational simulation is made to illustrate the efficiency of the algorithm based on block registration. Finally, we conclude our paper in section 5 .

\section{Restoration algorithm}

\subsection{Degradation model.}

The block diagram for general degradation model is shown in Fig.1. The degradation process is modeled as a degradation function, which together with an additive noise term, operates on an input image $f(x, y)$ to produce a degraded image $g(x, y)$. If $\mathrm{H}$ is a linear, position-invariant process, then the degraded image model is given in the spatial domain by Eq.1 or in frequency domain by Eq.2.

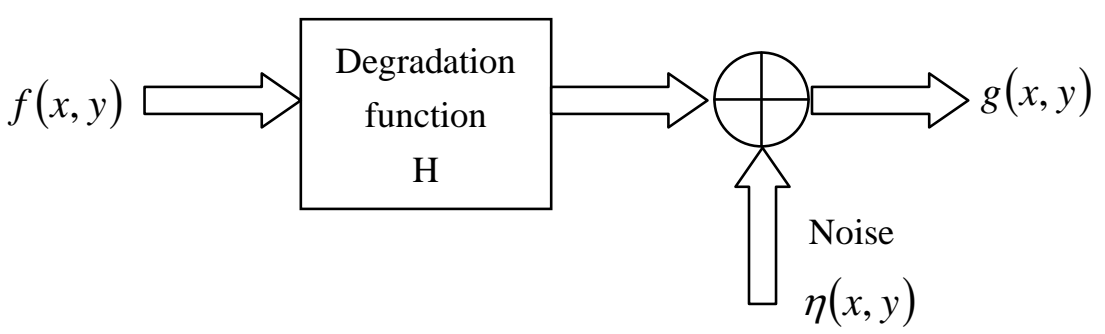

Fig.1: A model of the image degradation

$$
\begin{gathered}
g(x, y)=h(x, y) * f(x, y)+\eta(x, y) \\
G(u, v)=H(u, v) F(u, v)+\eta(u, v)
\end{gathered}
$$

Where $\eta(x, y)$ is the additive noise term, $h(x, y)$ is called as Point Spread Function (PSF).A true image $f(x, y)$ is estimated from a degraded image $g(x, y)$ based on prior knowledge of $\operatorname{PSF} h(x, y)$ and the statistical properties of noise $\eta(x, y)$. 


\subsection{Estimating the degradation function.}

A mathematical model of motion blur will be derived. An image is blurred by uniform linear motion between the image and the sensor during image acquisition. Image $f(x, y)$ is in planar motion. Assuming shutter opening and closing takes place instantaneously, and optical imaging process is perfect, not affected by image motion. If $\mathrm{T}$ is the duration of the exposure, and image $g(x, y)$ is blurred image. The blurred image is obtained by Eq.3.

$$
g(x, y)=\int_{0}^{T} f\left[x-x_{0}(t), y-y_{0}(t)\right] d t
$$

Where $x_{o}(t)$ and $y_{o}(t)$ are the time varying components of motion in the $x$-direction and $y$-direction. The Fourier transform of $g(x, y)$ is obtained by Eq.4. By defining $H(u, v)=\int_{0}^{T} e^{-j 2 \pi\left[u x_{0}(t)+v y_{0}(t)\right]} d t$, equation 5 is derived. $H(u, v)$ can be obtained if $x_{o}(t)$ and $y_{o}(t)$ are known.

$$
\begin{gathered}
G(u, v)=F(u, v) \int_{0}^{T} e^{-j 2 \pi\left[u x_{0}(t)+v y_{0}(t)\right]} d t \\
G(u, v)=H(u, v) F(u, v)
\end{gathered}
$$

Suppose that the image in question undergoes uniform linear motion in the ${ }^{X}$ - direction only, at a rate given by $x_{o}(t)=a t / T$ then the degradation function becomes Eq.6.

$$
H(u, v)=\frac{T}{\pi u a} \sin (\pi u a) e^{-j \pi u a}
$$

If we allow the $y$-component to wary as well with the motion given by $y_{o}(t)=b t / T$ then the degradation function becomes Eq.7.

$$
H(u, v)=\frac{T}{\pi(u a+v b)} \sin [\pi(u a+v b)] e^{-j \pi(u a+v b)}
$$

\subsection{An image restoration method based on block registration.}

Motion blur of target area is lesser and limited in intelligent video surveillance. Using the overall offset estimation method to recover, the result is bad and large amount of calculation. An image restoration method based on block operation is proposed in the paper. Blurred blocks are recovered, and clear blocks are not operated. The method can not only enhance the local information, but also not affect the overall image quality. 


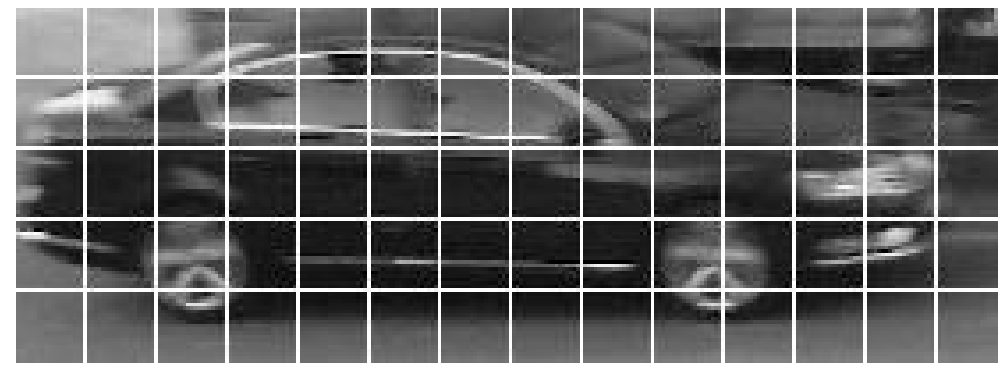

(a) Original image

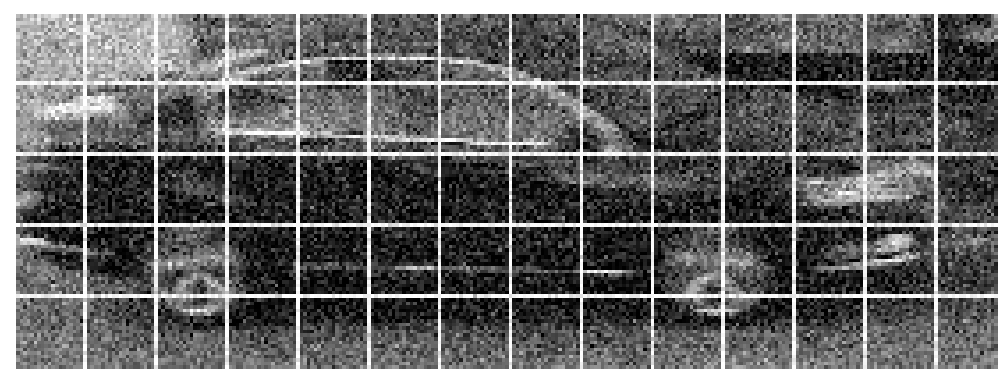

(b) Blurred image

Fig.2: Block operation of the image

The determine method is: the image $m \times n$ is divided into many sub-blocks of size of $\frac{m}{l} \times \frac{n}{l}$ pixels. Correlation matching algorithm is carried between each sub-block of original image and blurred image. According to the correlation coefficient, blurred sub-block in motion blurred image is found. The Wiener filtering is used for image restoration. The Wiener filter in Fourier domain can be expressed as follows Eq.8.

$$
H_{W}(u, v)=\frac{H^{*}(u, v)}{|H(u, v)|^{2}+S_{n}(u, v) / S_{f}(u, v)}
$$

Where $S_{f}(u, v)=|F(u, v)|^{2}, S_{n}(u, v)=|N(u, v)|^{2}$ are respectively power spectra of the original image and the additive noise, and $H(u, v)$ is the blurring filter. 


\section{Evaluation criteria}

The term peak signal-to-noise ratio (PSNR) is an expression for the ratio between the maximum possible value of a signal and the power of distorting noise that affects the quality of its representation. For the following implementation, let us assume we are dealing with a standard 2D array of data or matrix. The dimensions of the correct image matrix and the dimensions of the degraded image matrix must be identical. The mathematical representation of the PSNR is expressed by Eq.9. Where the MSE (Mean Squared Error) is calculated by Eq.10, m represents the numbers of rows of pixels of the images and $n$ represents the number of columns of pixels of the image.

$$
\begin{gathered}
\text { PSNR }=20 \log _{10}\left(\frac{M A X_{f}}{\sqrt{M S E}}\right) \\
M S E=\frac{1}{m n} \sum_{0}^{m-1} \sum_{-}^{n-1}\|f(x, y)-\hat{f}(x, y)\|^{2}
\end{gathered}
$$

The Root Mean Square Error (RMSE) is a frequently used measure of the difference between values predicted by a model and the values actually observed from the environment that is being modeled. A smaller value calculated for RMSE means that the image is of good quality. RMSE measure between the original image and the restoration image is given by Eq.11. The original image is represented as $f(x, y)$, restoration image $\hat{f}(x, y)$. The value of RMES is smaller, the effect of restoration image is better.

$$
R M E S=\sqrt{\frac{1}{m \times n}\left[\sum_{x=0}^{m-1} \sum_{y=0}^{n-1}[\hat{f}(x, y)-f(x, y)]^{2}\right]}
$$

\section{Experimental results and analysis}

The original image is shown in Fig.3, which is a car in the intelligent transportation field. Regardless of the blurred image in the degradation of the image block size and number is shown in Fig.4, the restoration algorithm based on block registration can improve the quality of the image on the vision. For video surveillance images in the field of intelligent transportation, interested area such as license plate and face often is smaller, and has less amount of calculation, thus ensuring the efficiency of the algorithm has higher recovery in shown in Fig.5. 


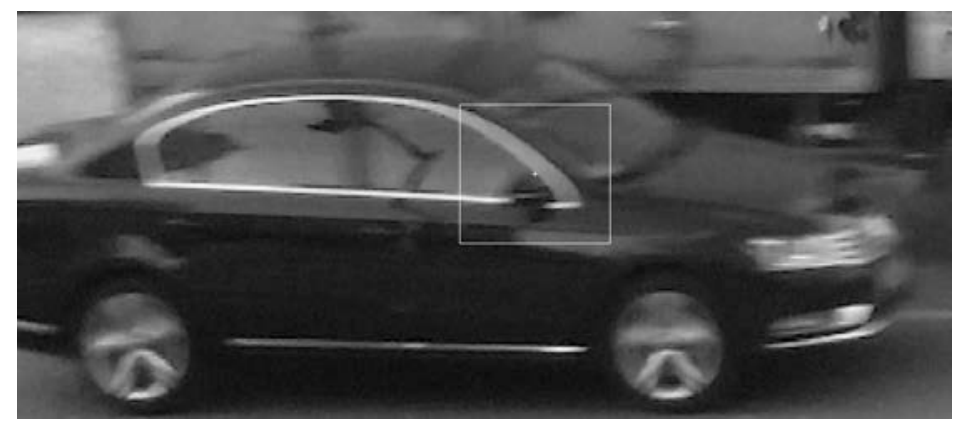

Fig.3: Original image

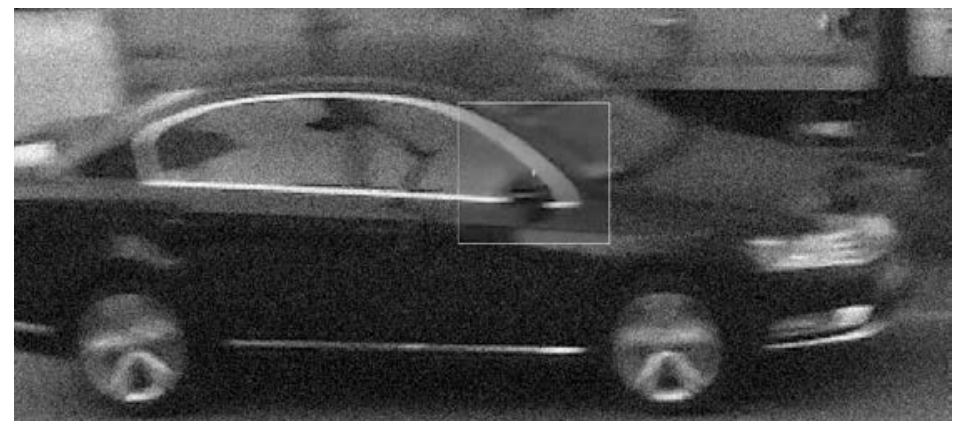

Fig.4: Blurred image

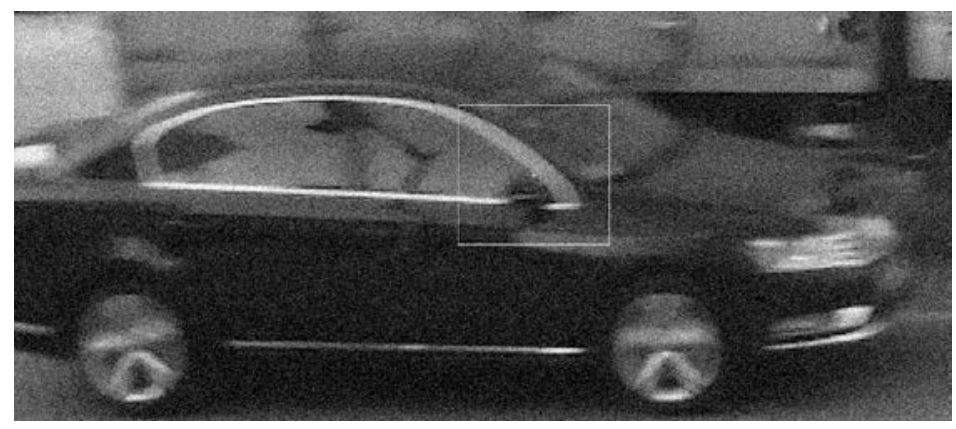

Fig.5: Restoration image

Through computing values of PSNR and RMES, the effects of images restoration are evaluated and compared in Table.1. The proposal is that the higher the PSNR, the better degraded image has been reconstructed to match the original image and the better the reconstructive algorithm. The smaller the RMES value is, the better the restoration effect is. Shown as Table.1, the new image restoration method based on block registration is good. 
Table 1: Comparing the performance of recovery

\begin{tabular}{|l|l|l|l|}
\hline No. & Performance & Blurred Image & Restoration Image \\
\hline 1 & PSNR & 53.5427 & 62.8956 \\
\hline 2 & RMSE & 0.05641 & 0.01786 \\
\hline
\end{tabular}

\section{Conclusions}

In order to improve the efficiency of image restoration, the image restoration of motion blurred images is studied and analyzed. Firstly, a model of the image degradation is introduced. Secondly, the degradation function is estimated. Finally, the new method of block registration combination wiener filter is proposed in order to make up for the inadequacy of wiener filter method. By MATLAB simulation, restoration images and performance of recovery are gained. Through the analysis of results, the presented method in this paper is better and accurate.

\section{Acknowledgements}

This work was financially supported by scientific research fund of Jilin provincial education department (2014, the number: 302), Project name is "Research on the key technology of portable target recognition system based on multi-sensors fusion”.

\section{References}

[1] Tao Xun. A motion-blurred image restoration algorithm based on block registration, Computer Applications and Software. Vol. 28, pp. 246-249, 2011.

[2] Wu Xianjin and Wang Runsheng, Regularized Image Restoration Based on Edge Restoration and Artifacts Removing, Journal of Electronics \& Information Technology, Vol. 28, pp. 577-580,2006.

[3] Chan T F, Shen Jianhong. Image Processing and Analysis[M]. Philadelphia: Society for Industrial and Applied Mathematics, 2005.

[4] Nagy J G, Palmer K, Perrone L. Iterative methods for image de-blurring: a Matlab object-oriented approach[J]. Numerical Algorithms, Vol.36, pp.73-76, 2004.

[5] Lam E Y. Blind bi-level image restoration with iterated quadratic programming [J].IEEE Trans Circuits and Systems-II: Express Briefs, Vol. 54, pp. 52-56,2007. 
[6] Tanaka M,Yoneji K,Okutomi M. Motion blur parameter identification from a linearly blurred image[A].Piscataway: IEEE, pp. 1-2, 2007.

[7] Le Xiang, Cheng Jian, and Li Min. Improved approach to motion blur identification based on random transform[J] infrared and laser engineering, Vol.40(5), pp. 963-969, 2011.

[8] Guo Yongcai, Guo Ruirui, and Gao Chao. Identification of blur parameters from motion blurred image[J].Chinese Journal of Scientific Instruments, Vol.31(5), pp. 1052-1057, 2010.

[9] Xie Wei and Qin Qiangqing. Estimating blur parameters of point spread function of motion-blurred image based on cepstrum[J]. Geometrics and Information Science of Wuhan University, Vol.33(2),pp. 128-131 2008.

[10] Li Yucheng,Jia Baohua, and Yang Guangming. Blur parameter identification and restoration of motion blurred image[J].Computer Engineering and Design, Vol.30(19), pp.4247-4249,2010. 